\title{
Upper Limb Multifactorial Movement Analysis in Brachial Plexus Birth Injury
}

\author{
Jorg Bahm ${ }^{1}$ \\ ${ }^{1}$ Franziskushospital, Euregio Reconstructive Microsurgery Unit,
Aachen, Germany
}

Address for correspondence Jorg Bahm, MD, PhD, Franziskushospital, Aachen 52074, Germany (e-mail: jorg.bahm@belgacom.net).

J Brachial Plex Peripher Nerve Inj 2016;11:e1-e9.

\begin{abstract}
Keywords

- motion analysis

- upper limb

- brachial plexus birth injury

- medial rotation contracture

Multifactorial motion analysis was first established for gait and then developed in the upper extremity. Recordings of infrared light reflecting sensitive passive markers in space, combined with surface eletromyographic recordings and/or transmitted forces, allow eclectic study of muscular coordination in the upper limb. Brachial plexus birth injury is responsible for various patterns of muscle weakness, imbalance, and/or simultaneous activation, soft tissue contractures, and bone-joint deformities, leading to individual motion patterns and adaptations, which we studied by means of motion analysis tools. We describe the technical development and examination setup to evaluate motion impairment and present first clinical results.

Motion analysis is a reliable objective assessment tool allowing precise pre- and postoperative multimodal evaluation of upper limb function.

Level of evidence: II.
\end{abstract}

\section{Introduction}

Brachial plexus birth injury (BPBI) is a severe nerve injury at the level of the brachial plexus, with individual outcome dependent on the severity of nerve root injury, incidence, and amount of microsurgical nerve reconstruction and secondary reconstructive procedures. ${ }^{1-5}$ Muscle weakness and simultaneous activation of antagonists, growth impairment of the affected limb, and soft tissue contractures are main factors explaining progressive motion impairment.

In the clinical course and especially for pre- and postoperative assessment, evaluation of passive and active range of motion (ROM) is mandatory and may be combined with muscle strength estimation using the British Medical Research Council grading from M1 to M5. These assessments are subjective, vary between observers, and are not easily repeated in little children. There is thus need for objective tools, also exploring biomechanical aspects of upper limb motion and providing datasets for offline analysis and reliable interpretation.

\section{Material and Methods}

The Progressive Development of Measurement Tools To coordinate clinical and biomechanical knowledge, we cooperate since 1996 on a regular basis with the laboratory for movement analysis at the Institute of Applied Medical Engineering (Helmholtz Institute) at the RWTH Aachen University, Germany (Director: Professor Dr. T. Schmitz- Rode) within the Department of Rehabilitation and Prevention Engineering (Head: Professor Dr. C. Disselhorst-Klug). Patients with upper limb movement impairment (and especially children with a $\mathrm{BPBI})$ were addressed over the years for specific noninvasive electromyography, named high spatial resolution surface EMG (HSR-EMG), ${ }^{6}$ to study the reinnervation pattern in the biceps muscle. Biceps-triceps co-contractions were then analyzed before and after treatment with botulinum toxin. ${ }^{7}$ received

May 29, 2014

accepted

January 22, 2016
DOI http://dx.doi.org/

10.1055/s-0036-1579762. ISSN $1749-7221$.
Copyright $\odot 2016$ Georg Thieme Verlag

KG Stuttgart · New York
License terms

(요 (1) $\odot$ 
When the movement analysis was expanded from lower limb gait analysis to the upper limb, ${ }^{8}$ we progressively applied this technology to record upper limb movements and extended the measurements to various parameters such as ROM and key muscle electromyograms.

Since 2005, our experience with the analysis of upper limb movements using the Vicon technology ${ }^{2}$ has been applied to a prospective group of 19 compliant children (age: 6-13 years; median: 10 years) suffering from BPBI complicated by a rotational shoulder imbalance with medial rotation contracture (MRC). These patients were selected according to their deformity (BPBI and MRC), their good compliance and age above 6 years, and their close residency to perform repetitive measurements. Reference data from six healthy volunteers (age: 15-24 years; median: 20 years) were included.

Since 2010, a new prospective group of so far four brachial plexus birth injury (BPBI) children with MRC of the shoulder has been added. The aim now is to investigate forces and torques acting on the joints, especially the glenohumeral joint (GHJ). Therefore, we started to include pre- and postoperative measurements of shoulder forces and torques before and after shoulder release surgery in children presenting different degrees of severity of shoulder MRC.

\section{Motion Recording Tool}

The Vicon technology is derived from a cinematographic tool. Our cooperation laboratory uses a motion-capturing system

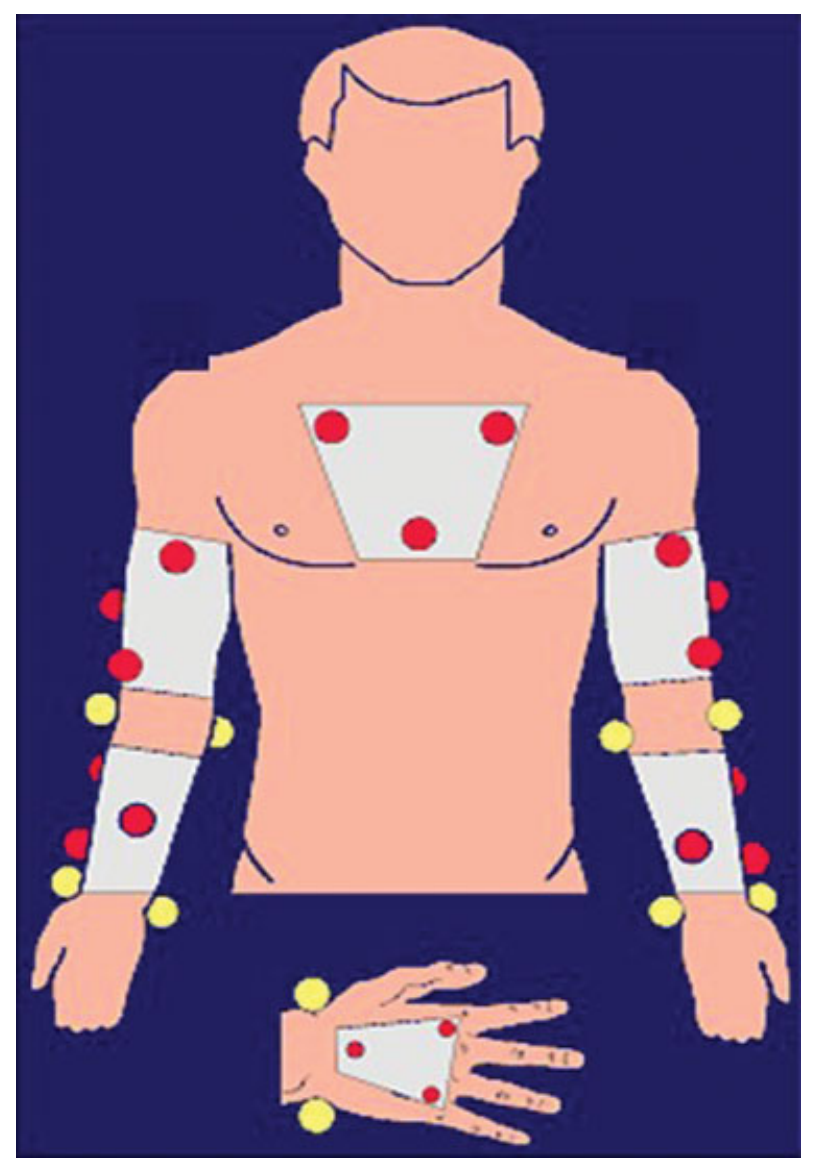

Fig. 1 Surface markers: positions on the chest and upper limb, tridimensional marker shape.

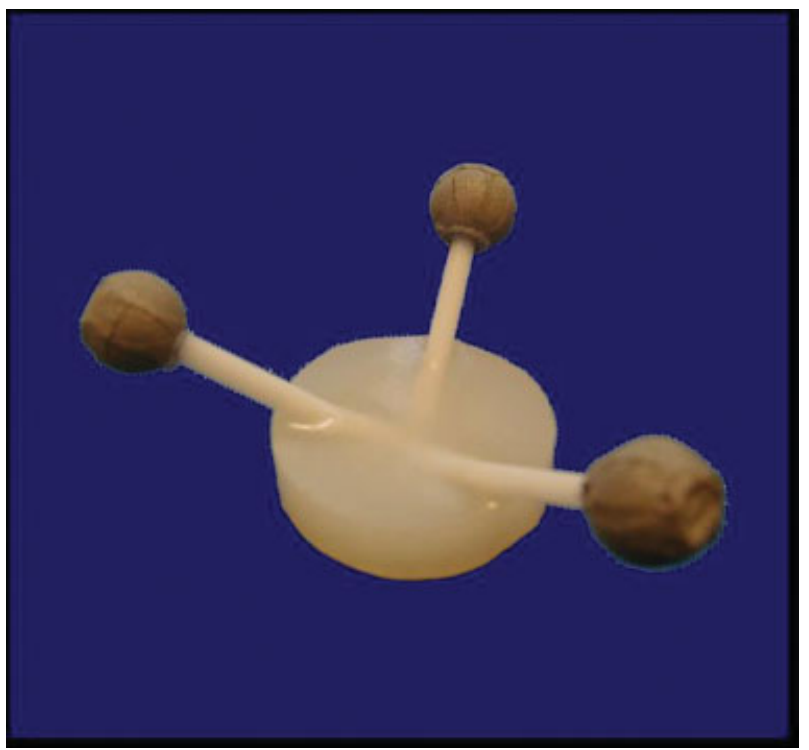

Fig. 2 Tridimensional coordinate system at different joint levels of the upper limb.

made of 10 infrared video cameras, including 32 analog and 16 eletromyographic (EMG) channels to track passive surface markers in space and time (-Figs. 1 and $\mathbf{3}$ ). The markers are placed on the upper limbs and the chest of the child according to standardized recommendations. ${ }^{7}$

The variety of motions that can be captured by the system is theoretically unlimited, but further investigation of the captured motion is limited by the availability of biomechanical models supporting the movement analysis.

\section{Biomechanical Models}

They are either kinematic or kinetic. Kinematics studies the movement of objects in space, according to their path, velocity, and acceleration, without considering the forces responsible for the movement. A kinematic model thus allows the measurement of joint angles, velocity, and acceleration. Kinetics studies the movement of objects accelerated by forces. A kinetic model thus enables the additional calculation of forces and moments (torques) applied onto the joints, and also inherent work, power, and energy.

The kinematic model used in our cooperation laboratory is based on the rigid body approach ${ }^{9,10}$ : each limb segment is assigned to one bone or group of bones (collar bone, scapula, humerus, forearm, hand). Motion between the segments is assumed possible only within the defined joints as a sheer rotation (no translation). - Fig. 1 shows the segmentation of the kinematic model and the marker configuration, with segment markers and temporary joint markers (the latter were applied during a static calibration trial to avoid erroneous data caused by skin movement).

The segment markers form a triplet where the marker extremities form a triangle and thus define one plane. Using these triplets allows an assessment of the motion of each segment in all six degrees of freedom. The rotational center of the shoulder joint has to be estimated as it is located deep under the skin surface. We assume that it lies at a certain distance 
below the acromion. This distance is assessed individually by a reference measurement for each patient. ${ }^{9}$ The shoulder joint is assumed to work like a ball and socket joint. This model is simple, with some inherent errors, but it allows us to study shoulder motion in daily activities, with or without external load, in a virtually complete ROM. After determination of the joint coordinate system for each joint, the joint motions can be described as relative rotations between these coordinate systems; thus, the kinematic model is completely defined.

\section{From Kinematics to Kinetics}

The further developed kinetic model to calculate forces and torques is based on the concept of inverse kinematics used in robotics. Inverse kinematics (or inverse dynamics, or reverse transformation) applied onto an industrial robotor allows the calculation of joint angles of the robotic arm segments based on the position and orientation of the tool center point, that is, the end effector. Inverse kinematics is the counterpoint to forward kinematics. The last element of the kinematic chain, the so-called end effector, is moved into the desired position. The other segments must adapt considering the degrees of freedom of their joints. The human arm with its joints also represents a kinematic chain. When we position our hand, the other upper limb joints move into a determinated position; the relative angles may be calculated by inverse kinematics. Difficulties in assessment may arise as there may be different configurations (for a given end position of the tool center point) or forbidden ones (mathematically correct but unrealistic for the joint positioning). The solution of inverse dynamic problems is achieved by algebraic, geometric, or numeric methods. The algebraic method allows the calculation of a homogenous matrix describing the position and orientation of the end effector by a repeated reversal of the Denavit-Hartenberg transformation matrices. Denavit and Hartenberg described a system of conventions, parameters, and transformations ${ }^{11}$ defining frames of reference in robotic application. The convention is based on a reference system in space, where the z-axis is defined as being in line with the joint axis (see below). The $\mathrm{x}$ and $\mathrm{y}$-axes are defined accordingly to create a right-handed coordinate system. When using inverse kinematics algorithms, forces and torques at the level of the different joints may be calculated. We concentrate on the glenohumeral joint and its tridimensional coordinate system (-Fig. 2).

Each coordinate axis is defined as the rotation axis for the concerned movement. In the shoulder, the x-axis concerns flexion and extension ( $F_{\mathrm{x}}$ is perpendicular to the flexionextension plane); y-axis concerns abduction and adduction ( $F_{\mathrm{y}}$ is perpendicular to the abduction-adduction plane); and $z$-axis concerns the lateral and medial rotation, identical with the humeral axis ( $F_{z}$ is perpendicular to the rotational plane).

Without any external force, the forces applying in the different directions $F_{\mathrm{x}}, F_{\mathrm{y}}$, and $F_{\mathrm{z}}$ now may be calculated. If an external force is applied (like when lifting a charge), a force transducer is necessary to integrate these additional forces into the determination of $F_{\mathrm{x}}, F_{\mathrm{y}}$, and $F_{\mathrm{z}}$. In our patient series with MRC, we studied both. The force transducer converts measured forces into output signals, further sent to data acquisition systems like computers. We used a Schunk FT Gamma transducer, configuration SI-130-10 (Schunk GmbH \& Co. KG; Lauffen Germany, www.schunk.com).

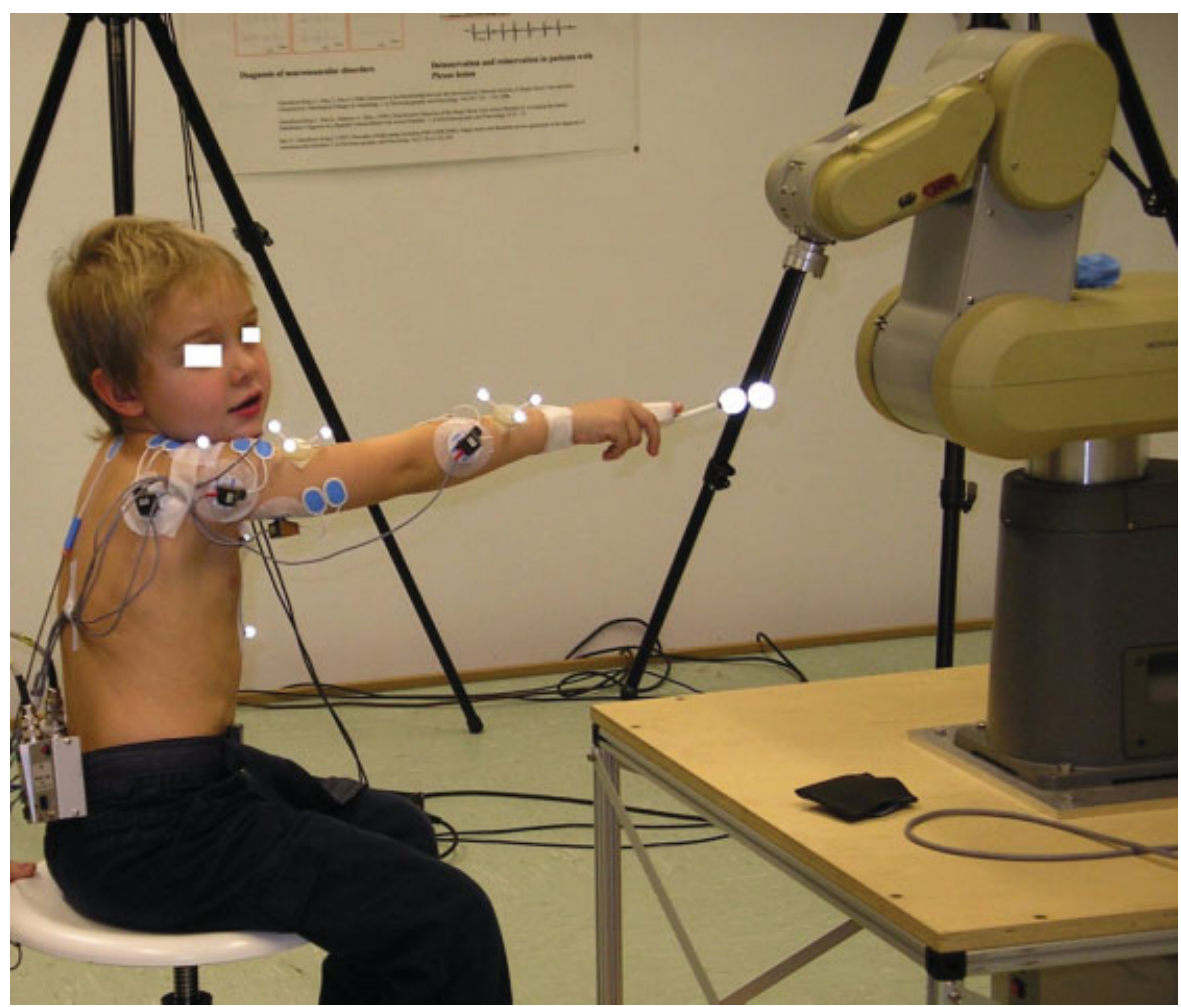

Fig. 3 Examination setting with the skin markers and the robot. 
Table 1 Samples in shoulder motion analysis

\begin{tabular}{|l|l|l|}
\hline Type of exam & Patients & Volunteers \\
\hline Robot & 14 & 3 \\
\hline Curves & 13 & 3 \\
\hline Figure of eight & 10 & 2 \\
\hline Straight line & 10 & 2 \\
\hline Parking ticket & 4 & 5 \\
\hline Lateral rotation & 3 & 0 \\
\hline Hand to neck & 2 & 1 \\
\hline Abduction-rotation & 1 & 0 \\
\hline
\end{tabular}

The forces mainly correspond to the deforming pressures onto the joint surfaces. Muscle strength in the examined extremity muscles (which all act through a rotational movement around a joint center) is better represented by the measured torque or lever arm (force $\times$ lever arm $=$ torque).

\section{Forces onto the Glenohumeral Joint}

Applying these concepts to our MRC patients, we focused on $\mathrm{GHJ}$ deformation and thus looked at uncompensated or excessive negative $F_{\mathrm{x}}$ (compression onto the glenoid) and negative $F_{\mathrm{y}}$ forces (retropulsion of the humeral head) in the resting arm (adducted along the chest), and negative $F_{\mathrm{x}}$ and positive $F_{\mathrm{z}}$ in the abducted arm (rotation of the coordinate axes). One has to consider that the axes change through any upper limb motion, as any new position of the limb changes the predefined axes of motion direction. When applying the coordinate system to the right or left shoulder, one has to consider that only $F_{\mathrm{x}}$ changes his positive/negative value from right (basic definition) to left; the two other orientations $\left(F_{\mathrm{y}}\right.$ and $\left.F_{\mathrm{z}}\right)$ remain the same.

\section{Motion Patterns}

The child is asked to perform a standardized movement (for our study purpose, we initially concentrated on a movement from a position of full medial rotation of the shoulder with an adducted arm to a full lateral rotation [LR] with an abducted arm). As not all motion patterns are recorded entirely by the Vicon system due to marker superposition, some segmented simple movements were necessary to develop a routine examination algorithm (figure of eight, straight line, spiral movement, rotation in the $y-z$ plane; - Table 1). ${ }^{12}$ To ensure a reproducible movement, the present examination is always performed with a robotic arm (Mitsubishi Movemaster EX model RV M2; - Fig. 3) responsible for a standardized movement. ${ }^{12-14}$ Both the movements of the arm and that of the markers in space are recorded. As the cameras are placed in the laboratory at standardized locations and record the arm from different observer angles, combination of the data allows threedimensional reconstruction. Specific software allows to observe the recorded motion sequence and the movement of the markers in space, and to calculate time-related curves of the different movements (shoulder abduction and adduction, flexion and extension, lateral and medial rotation). Depending on the study subject, the movement (ROM) analysis is further completed by concomitant recording of surface EMG of selected target muscles, using surface electrodes on standardized positions (defined in our laboratory by the SENIAM recommendations, related to the SENIAM project [Surface ElectroMyoGraphy for the Non-Invasive Assessment of Muscles], a European concerted action in the Biomedical Health and Research Program [BIOMED II] of the European Union). External forces are measured using a handle force transducer ${ }^{13,14}$ and calculated through inverse dynamics transformation matrices.

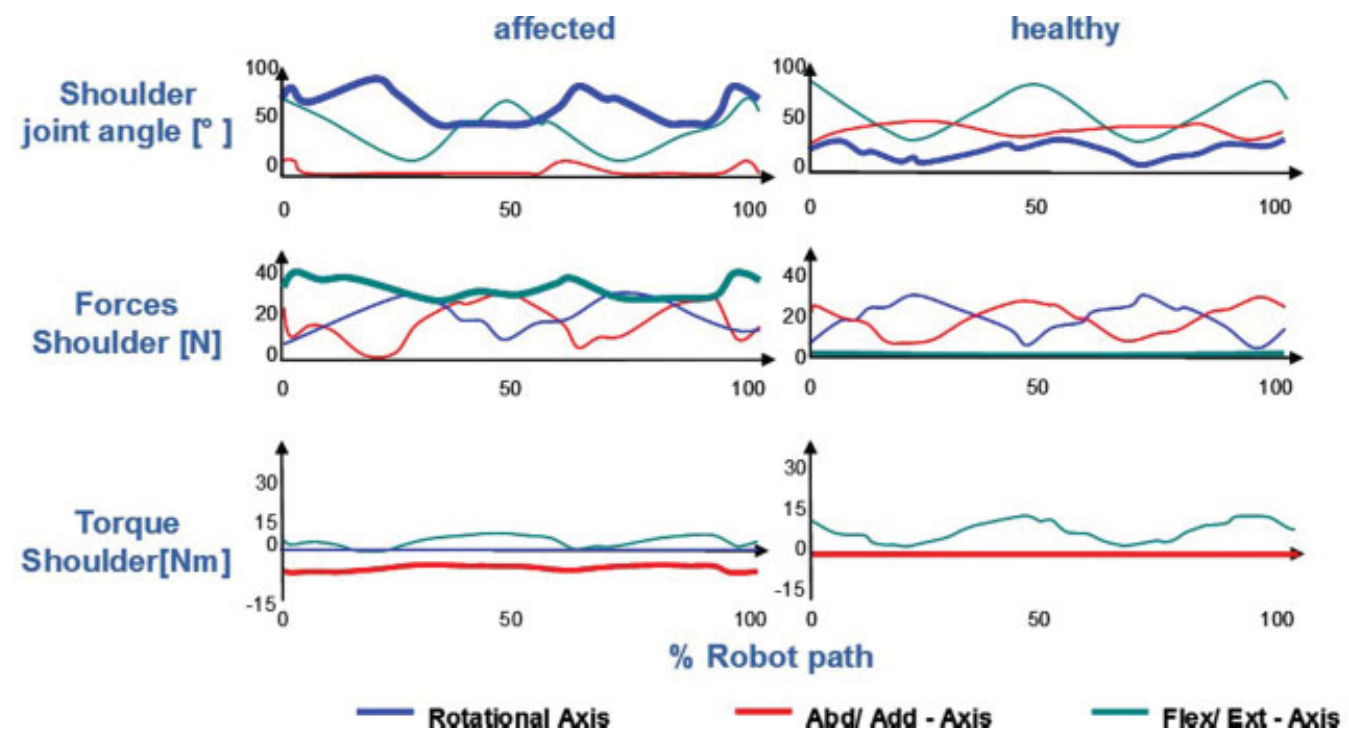

Fig. 4 Child with left BPBI and severe MRC shoulder deformity. The picture shows the positioning of the passive reflective markers and the EMG surface electrodes. The graph shows the ROM, forces, and torques on the affected and healthy side, according to the three motion axes of flexionextension ( $F_{x}$, green line), abduction-adduction ( $F_{y}$, red line), and rotation ( $F_{z}$, blue line). One observes the limitation of the rotation and the pathologic position of the affected humeral head with MRC (thick blue line, above left) and the major raise of $F_{x}$, measured positive on the affected left side, thus representing a force vector directed into the glenoid cavity with a potential deforming effect. $F_{\mathrm{y}}$ and $F_{\mathrm{z}}$ are comparable on both sides. 


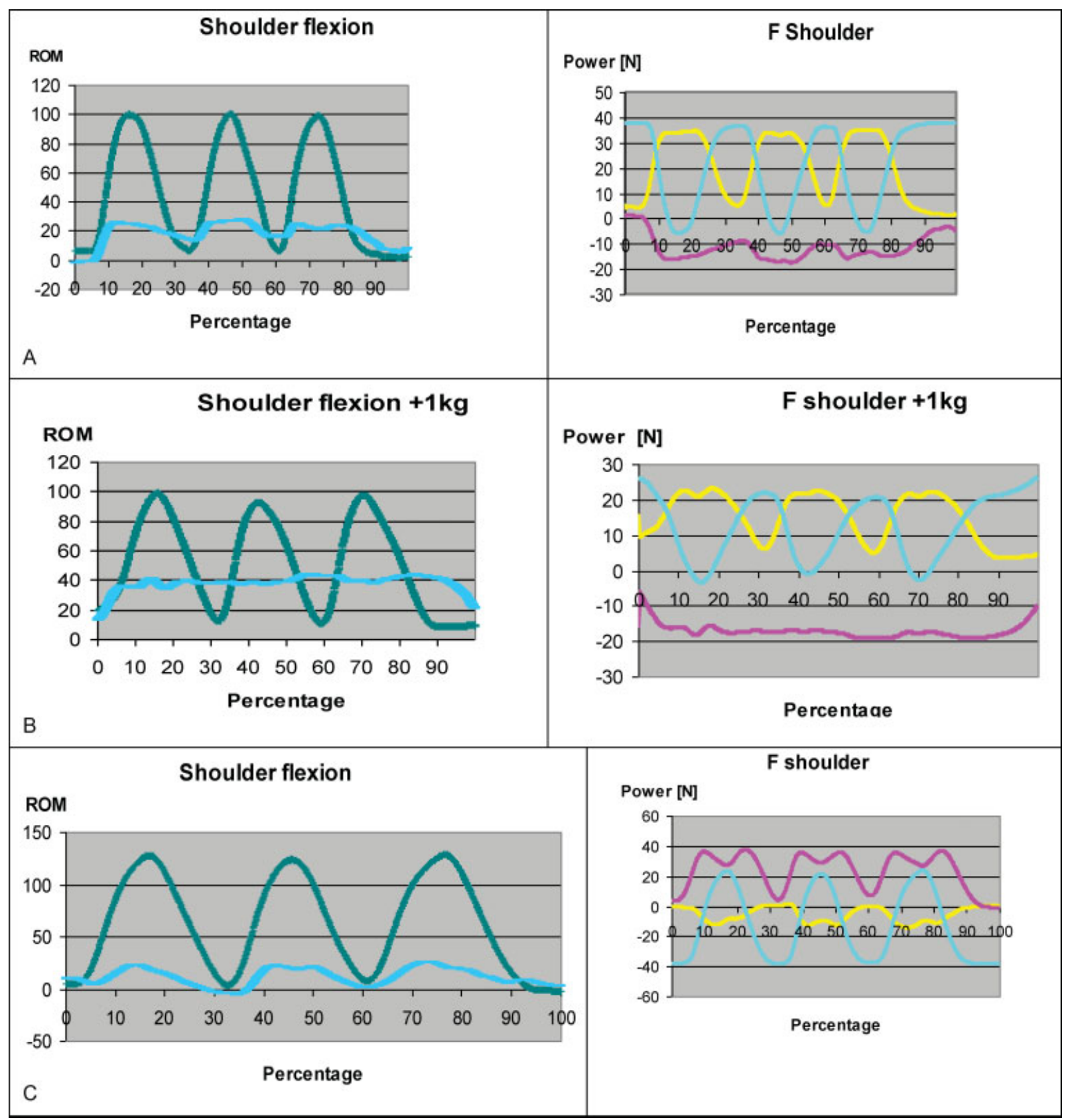

Fig. 5 Pre- and postoperative shoulder flexion and related forces. (a) (First row): preoperative situation of the affected side. ROM: green line $=$ flexion/extension, turquoise $=$ rotation; forces $F_{x}$ yellow, $F_{y}$ pink, $F_{z}$ turquoise. (b) (Second row): like (a), loaded with $1 \mathrm{~kg}$. (c) (Third row): preoperative situation of the healthy side. (All horizontal timelines called "percentage" represent the percentage of the accomplished robot pathway.) (d) (First row): like (c), but loaded with $1 \mathrm{~kg}$. (e) (Second row): postoperative status of affected side. (f) (Third row): like (e), but loaded with $1 \mathrm{~kg}$. (All horizontal timelines called "percentage" represent the percentage of the accomplished robot pathway.)

Results are presented as time-related curves showing ROM, muscle activity, forces, and torques ( - Fig. 4).

Children with variable severity of shoulder MRC present with different rest positions, as the neutral rotation position of the humeral head is progressively changed into a more medially rotated position ( - Fig. 4 ). As this affects the correct marker position and labeling procedure before any motion analysis, a corrective "off-set" factor corresponding to the severity of MRC had to be added in the MRC study group.

\section{Results}

\section{Recorded Measurements}

- Table 1 summarizes the type of measurements in 19 children suffering from BPBI and six healthy volunteers. In eight 


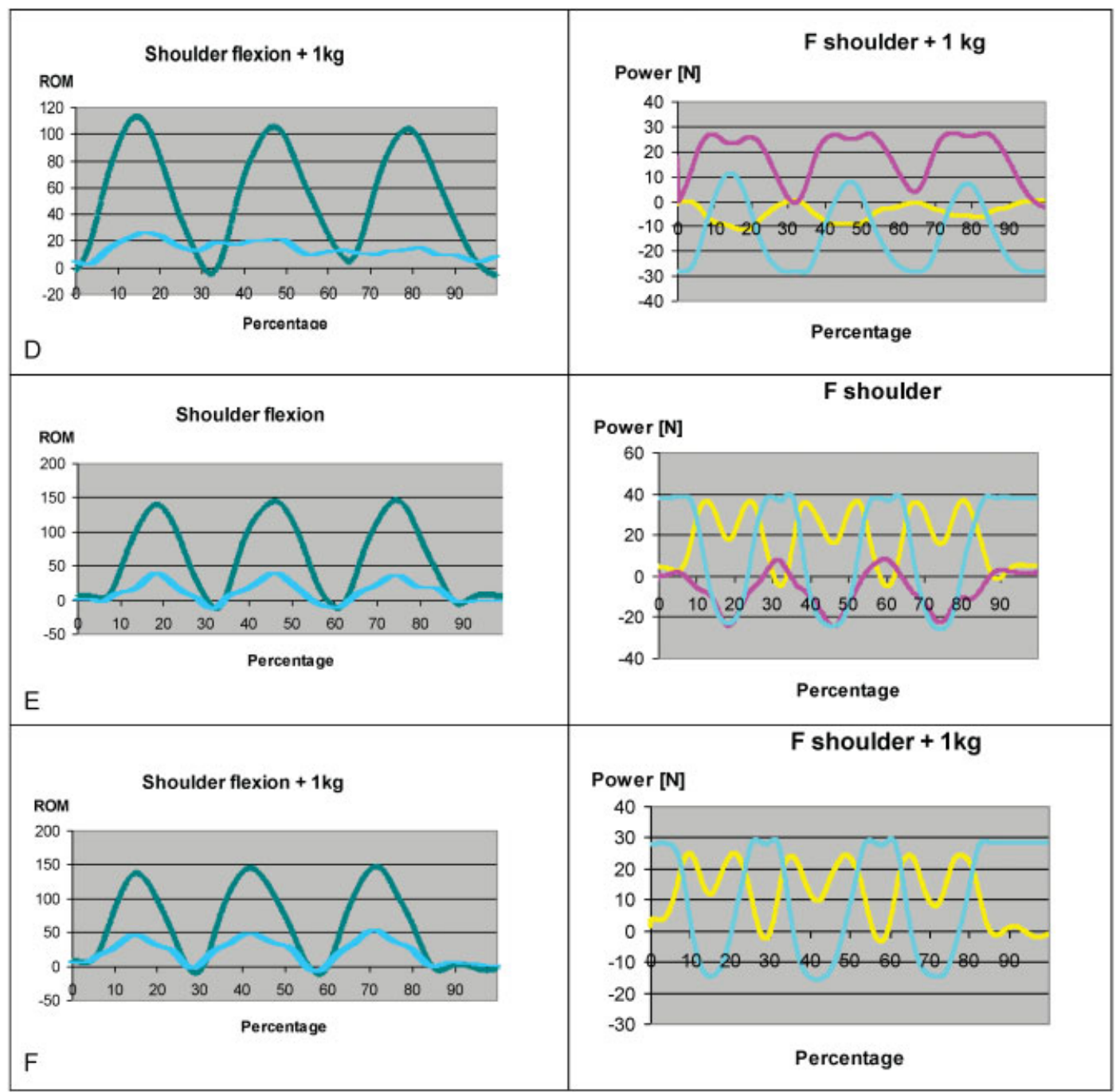

Fig. 5 (Continued)

patients, multiple measurements were made. Their examination was necessary to establish a standardized and validated evaluation routine both in healthy subjects ${ }^{12}$ and in obstetric brachial plexus lesion (OBPL) children.

There were numerous technical considerations and refinements over time. It was obviously necessary to adapt the robot to variable sizes of examined children and also to improve children's security while interacting with a machine processing a movement without any concern about the individual child's reactions. Also, the motion path had to be adapted to the anthropometric conditions of the examined children (a small child could not cope with very big movements and a tall child with very small movements ${ }^{14}$ ). Also, to estimate the forces, the complexity of the motion pattern had to be simplified.

-Fig. 4 shows an example of standardized and repetitive movement of the shoulder, as a result of a single plane flexion-extension tracking exercise following the path of the robot arm. The motion pattern of the healthy contralateral arm allows us to compare with reduced joint amplitudes and global pathway alteration.
In - Fig. 5, we present a pre- and postoperative examination (MRC treated by anterior release (AR), patient 1) focusing on shoulder flexion and the inherent forces and torques, reported as the timeline of percentage of the accomplished robot path (shortened to "percentage" in the graphs). The improved passive LR after surgery becomes obvious through the return to zero of the rotation line (compare $\mathbf{- F i g . 5 a}$ [preoperative], - Fig. $\mathbf{5 c}$ [the healthy side], and - Fig. 5e [after the release and improvement of passive LR with the arm adducted]). Also, the amplitude of shoulder flexion is increased. In each figure, we associate the repetitive shoulder movement (to simplify, we concentrated on one movement, here shoulder flexion) with the forces without and with an external load (a 1-kg weight hold in the hand). - Fig. 5a shows a peak "negative" $F_{x}$ (left shoulder), permanently slight negative $F_{\mathrm{y}}$, and positive $F_{\mathrm{z}}$-we emphasize the pathologic $F_{\mathrm{x}}$ and $F_{\mathrm{y} .}=$ Fig. 5b, with an external load, shows only a higher plateau for $F_{\mathrm{x}}$. In $\mathbf{- F i g . ~} \mathbf{5} \mathbf{c}$, the healthy right side shows a neutral $F_{\mathrm{x}}$ and a positive $F_{\mathrm{y}}$ pushing the head forward; the same appears under load (-Fig. 5d). After the anterior release surgery, $F_{\mathrm{x}}$ remains unchanged and $F_{\mathrm{y}}$ oscillates from slight negative to zero. 


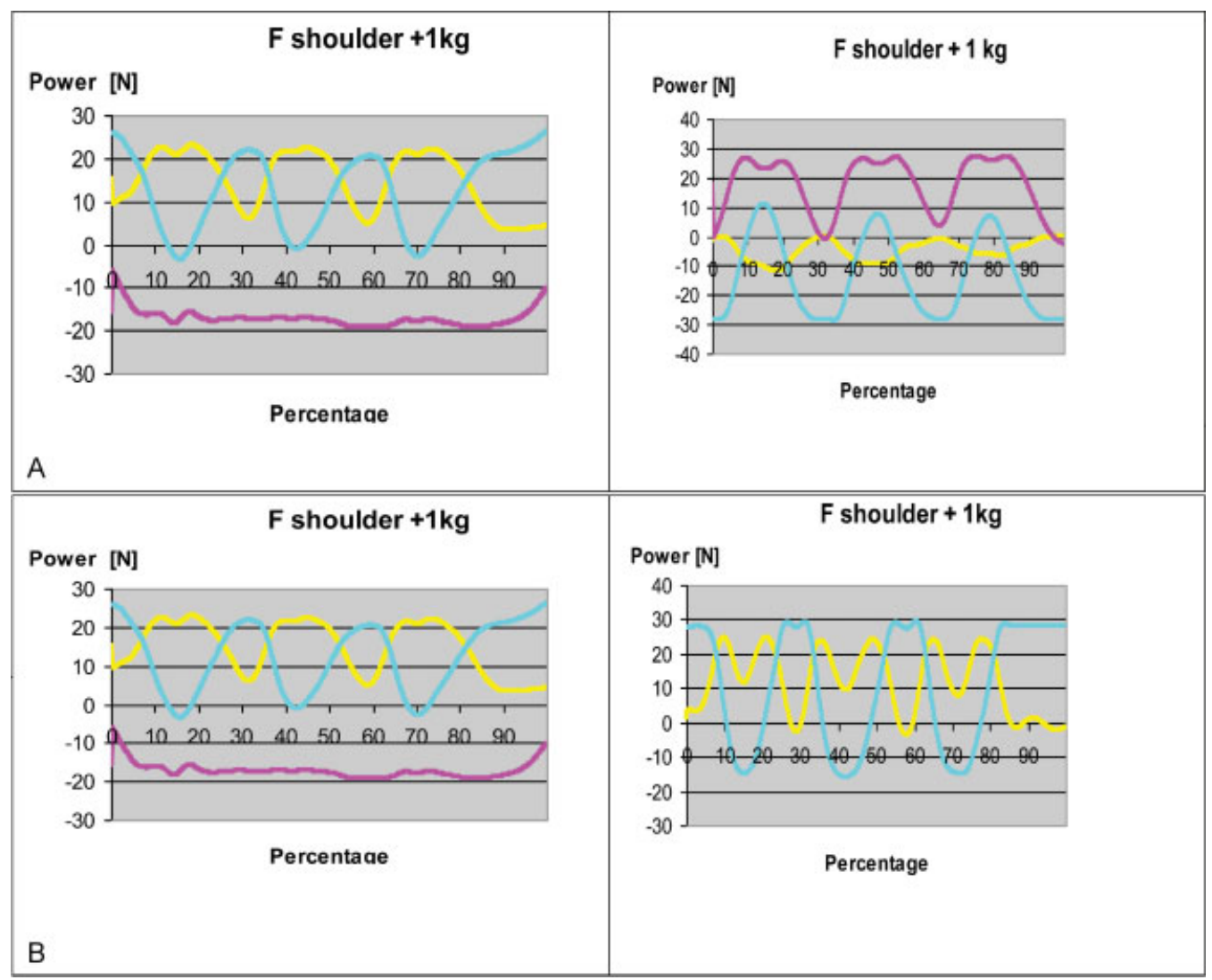

Fig. 6 (a) Same patient as in - Fig. 5. Significant comparative views showing the forces on the affected shoulder, loaded with $1 \mathrm{~kg}$ (left screen), compared with the healthy side (right screen). ("Percentage" means the accomplished percentage of the robot pathway). (b) Same patient as in - Fig. 5. Significant comparative views showing the forces on the affected shoulder loaded with $1 \mathrm{~kg}$ before (left screen) and after surgery (right screen). ("Percentage" means the accomplished percentage of the robot pathway.)

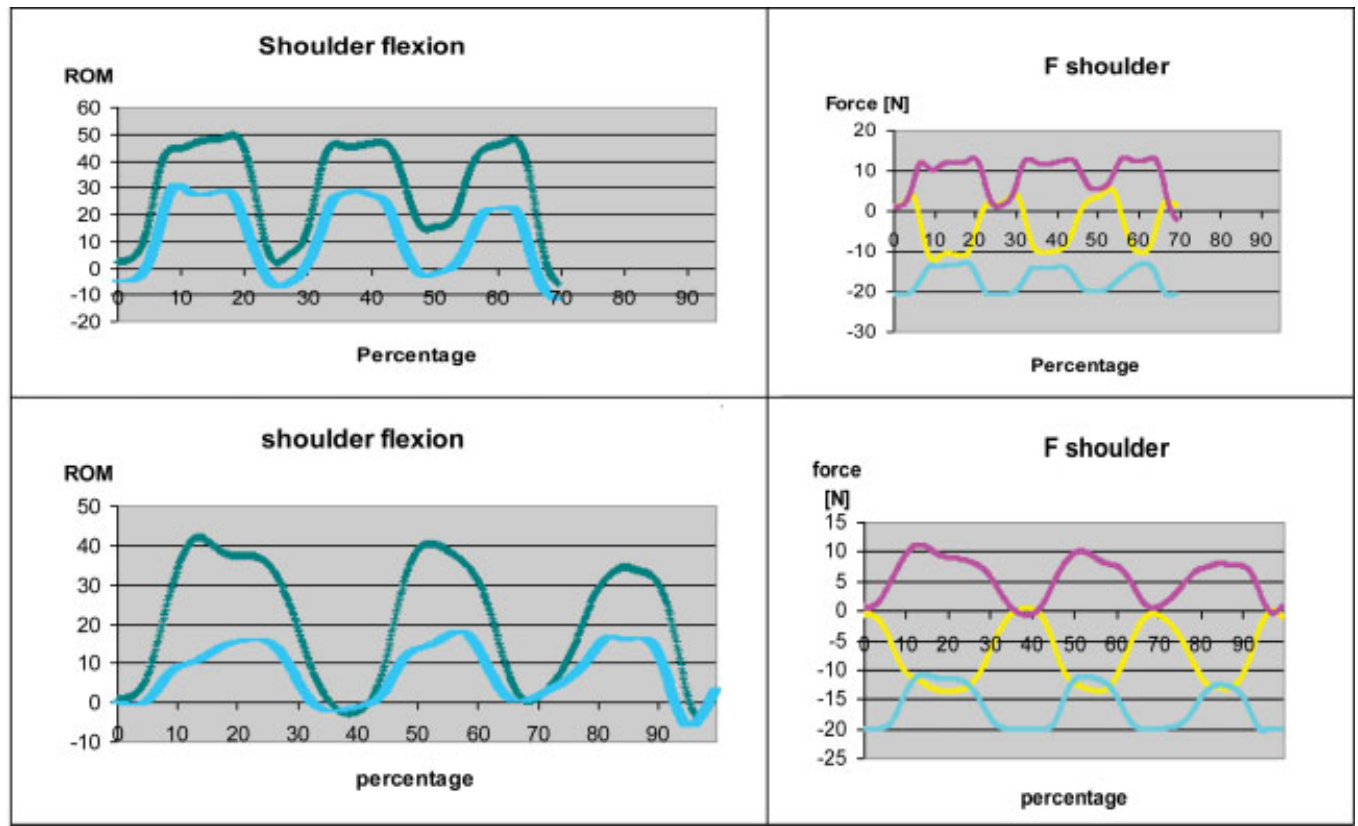

Fig. 7 Another patient with increased MRC. The two graphs represent the pre- (upper graph) and postoperative forces (lower graph) at the right affected shoulder. ("Percentage" means the accomplished percentage of the robot pathway.) 
Table 2 Measurements in BPBI children with MRC

\begin{tabular}{l} 
1. Left BPBI, 8 y, congruent joint \\
Pre-op: pLR(ADD), 0 degree (off-set 0 degree) \\
Post-op: pLR(ADD), 50 degrees \\
(results of measurement: - Figs. 5 and $\mathbf{6}$ ) \\
\hline 2. Right BPBI, 8 y, dorsal subdislocation of the \\
humeral head and dorsal pseudoglenoid \\
Pre-op: pLR(ADD), - 30 degrees (off-set 30 degrees) \\
Post-op: pLR(ADD), 25 degrees, maintained dorsal \\
subdislocation (results: - - Fig. 7 ) \\
\hline 3. Right BPBI, 11 y, dorsal subdislocation of the humeral \\
head and dorsal pseudoglenoid \\
Pre-op: pLR(ADD), -30 degrees (off-set 30 degrees) \\
No operation \\
4. Right BPBI, 15 y \\
Pre-op: pLR(ADD), - 50 degrees (off-set 50 degrees) \\
No operation
\end{tabular}

Note: The "off-set" value is a corrective item when the neutral position cannot be reached by the child and therefore a correction has to be integrated, equivalent to the negative pLR(ADD). pLR(ADD) means passive lateral rotation ROM with the arm adducted.

-Fig. 6 applies to the same patient's measurements and compares two important issues: in - Fig. 6a, one can observe the different forces applying to a healthy and (nonoperated) MRC shoulder under a standard shoulder flexion movement: Compared with the normal situation, the $F_{\mathrm{x}}$ component is enhanced (negative direction, as the left side is examined); $F_{\mathrm{y}}$ is permanently negative and $F_{z}$ oscillates at a higher force level. - Fig. $\mathbf{6 b}$ shows that anterior release surgery allows the $F_{\mathrm{y}}$ component to become less negative and even neutral again.

-Fig. 7 shows pre- and postoperative force changes in a second patient. One observes that the rotational component (turquoise line in the two left graphs) has diminished after surgery, but the force patterns remain unchanged. The dorsal subluxation of the humeral head has not been corrected by surgery, although the range of passive LR with the arm in adduction (pLR[ADD]) has been improved.

- Table 2 presents a synopsis of the measurements on BPBI children with MRC, both pre- and postoperatively, and the actual results on pathologic force components.

\section{Modelling}

As a mathematical simulation, we calculated the forces arising in a standardized shoulder flexion movement with different humeral head positions (increasing medial rotation). Inverse dynamics were applied to an ideal motion dataset and the resulting forces and torques were calculated. ${ }^{15}$ When a shoulder flexion is executed, and the medial rotation position increased, there is increasing negative $F_{\mathrm{x}}$, positive $F_{\mathrm{y}}$, and a sinusoidal decreasing negative $F_{z}$.

\section{Discussion}

We are not aware of other contributions analyzing movement patterns in children affected by BPBI. This type of examination thus might become in the near future the noninvasive, easy to perform, repetitive, and objective measuring tool to assess the functional status of an upper limb for diagnostic purposes, pre- and postoperative (comparative) assessments, and also for efficiency evaluation regarding treatment modalities like physiotherapy. Some prognostic factors also might be identified.

Nevertheless, motion analysis and its extensive setups and recordings require compliant children older than 4 years. As many treatment modalities in BPBI should be applied before 2 years, ${ }^{1}$ we still face the limitation of our inability to examine the children at the onset of muscle imbalance and pathologic forces onto the musculoskeletal system.

Another major problem regarding development and application of these tools is the different language spoken by medical clinicians and engineers. Our personal experience is made of a regular cooperation with biomedical engineers over 15 years, and yet expectations on both sides are not easily reached. Clinicians tend to consider the technical tools as being magic, responding easily to their questions about pathophysiology and biomechanics. Our investigation focused on pathological forces applied to the glenoid, and we expected a software showing force vectors active in a defined motion. The reality and common rationale has been described above and turned out to be much more complicated than believed initially: although we got some information about forces applied to one joint in one movement, along predefined coordinate axes, we are still unable to give a generalized view stating that a permanent force vector would be responsible over time for the $\mathrm{GHJ}$ deformation. On the other hand, we learned how any movement in space can be divided into motion vectors along three anatomical axes, allowing us to understand how a global upper limb movement executed in daily life activities would include more or less active lateral shoulder rotation on the affected and healthy side.

All motion patterns may be observed in a longer motion sequence and recorded data are ready for a remote analysis by independent objective observers.

Partner engineers must be constantly connected to clinical reality; bilateral conferences must become more regular. ${ }^{16}$ Motion analysis in the future will be much more than the initial video recording of movements, including angles, EMG signals, forces, or torques. It will develop into a multifactorial tool, coming up with a reference base of normal datasets, a hardware memory storing individual prior recordings, and a clear examination filter focusing on specific questions. The tool must exit research laboratories to become a regular evaluation tool in orthopedic or physiotherapeutic consultations, easy to handle, favoring repetitive recordings. Finally, the analysis should be paid for by the insurances.

The dream of "modelling" reconstructive surgery and studying the benefit on a computer, before even doing one incision, will remain such for some further years.

\section{References}

1 Bahm J. Obstetric brachial plexus palsy-clinics, pathophysiology and surgical treatment [in German]. Handchir Mikrochir Plast Chir 2003;35(2):83-97 
2 Bahm J. Secondary procedures in obstetric brachial plexus lesions [in German]. Handchir Mikrochir Plast Chir 2004;36(1):37-46

3 Bahm J, Becker M, Disselhorst-Klug C, et al. Surgical strategy in obstetric brachial plexus palsy: the Aachen experience. Semin Plast Surg 2004;18:285-299

4 Borschel GH, Clarke HM. Obstetrical brachial plexus palsy. Plast Reconstr Surg 2009;124(1, Suppl):144e-155e

5 Malessy MJA, Pondaag W. Nerve repair/reconstruction strategies for neonatal brachial plexus palsies. In: Chung KC, Yang LJS, McGillicuddy JE, eds. Practical Management of Pediatric and Adult Brachial Plexus Palsies. London: Elsevier; 2012:86-102

6 Disselhorst-Klug C, Bahm J, Ramaekers V, Trachterna A, Rau G. Non-invasive approach of motor unit recording during muscle contractions in humans. Eur J Appl Physiol 2000;83(2-3):144-150

7 Bahm J, Meinecke L, Brandenbusch V, Rau G, Disselhorst-Klug C. High spatial resolution electromyography and video-assisted movement analysis in children with obstetric brachial plexus palsy. Hand Clin 2003;19(3):393-399

8 Rau G, Disselhorst-Klug C, Schmidt R. Movement biomechanics goes upwards: from the leg to the arm. J Biomech 2000;33(10):1207-1216

9 Williams S, Schmidt R, Disselhorst-Klug C, Rau G. An upper body model for the kinematical analysis of the joint chain of the human arm. J Biomech 2006;39(13):2419-2429
10 Zatsiorsky VM. Kinetics of Human Motion. Champaign, IL: US Human Kinetics; 2002

11 Denavit J, Hartenberg RS. A kinematic notation for lower-pair mechanisms based on matrices. Trans ASME. J Appl Mech 1955; 23:215-221

12 van Oosterhout E. Using a robot in upper extremity movement analysis in children. Stagebericht faculteit der gezondheidswetenschappen, bewegingswetenschappen Universiteit Maastricht; Februari 2004

13 Popovic N, Williams S, Schmitz-Rode T, Rau G, Disselhorst-Klug C. Robot-based methodology for a kinematic and kinetic analysis of unconstrained, but reproducible upper extremity movement. J Biomech 2009;42(10):1570-1573

14 Popovic N. Modellbasierte Erfassung der dreidimensionalen Kinetik der Bewegung der oberen Extremitäten [dissertation]. Aachen: RWTH Aachen Elektrotechnische Fakultät; 2010

15 Kleiber T, Popovic N, Bahm J, Disselhorst-Klug C. A modeling approach to compute modification of net joint forces caused by coping movements in obstetric brachial plexus palsy. J Brachial Plex Peripher Nerve Inj 2013;8(1):10

16 Disselhorst-Klug C, Bahm J. An Update on Kinematic Evaluation of the Upper Extremity. Seminar of the Department of Orthopaedics and Traumatology, Erasme University Hospital, Brussels; 2009 